\title{
UN MODELO DE PRUEBA ESCRITA QUE REVELA CAPACIDADES RELACIONADAS CON EL PROCESO DE APRENDIZAJE
}

\author{
NIGRO, R.G. \\ Rua Baquirivu, 353. CEP 04404-030 São Paulo, S.P. Brasil.
}

\section{SUMMARY}

Despite the criticisms made by several teachers, the taxonomy originally proposed by Bloom et al and some of its most recent versions, remain largely used in the making of written tests.

By employing the concept of «Capability", types of questions for written tests are compared and distinguished with respect to the capabilities required to solve them. This distinction has proved useful for chosing questions that allow a probing of particular capabilities developed during the learning process. Further, in contrast to the use of Bloom's taxonomy, the choice of questions for written tests according to the criteria presented here has shown to be coherent with the recent idea of teaching science as an investigative process.

\section{INTRODUCCIÓN}

En la vida cotidiana de los profesores, la evaluación aún está desprovista de su dimensión realmente pedagógica, sirviendo más para seleccionar y clasificar que para retroalimentar las actividades docentes y de los alumnos (Rodríguez et al., 1992). Además de esto, entre aquéllos que realizan una evaluación formativa, un instrumento particular de evaluación merece ser discutido de mejor forma. Me refiero, específicamente, a las pruebas escritas, las cuales parecen aún no haber sufrido contribuciones más recientes y con tanta divulgación como las de discriminación de los asuntos por área en diferentes ítems de una taxonomía realizada por Bloom y sus colaboradores $^{1}(1956,1971)$.

Ya Bloom puede ser considerado como un conductista (Allal et aI., 1986). La taxonomía que él y sus colaboradores propusieron se preocupa esencialmente en «ser una clasificación de los comportamientos que representan resultados pretendidos del proceso educativo" (Bloom et al., 1956). En otras palabras, lo que se nos presenta en la taxonomía son los «comportamientos esperados -la forma en que los alumnos deben actuar, pensar o sentir, como resultado de su participación en alguna unidad de enseñanza» (Bloom et al., 1956), y no aspectos que se refieran al proceso de aprendizaje en sí.

De esta forma, las pruebas escritas basadas en la taxonomía de Bloom pueden, de hecho, retroalimentar una unidad de enseñanza (principalmente desde una perspectiva neoconductista) indicando qué comportamientos pretendidos o esperados el alumno consiguió alcanzar o no. Sin embargo, en esta perspectiva existe una limitación producto de la incapacidad de retroalimentar de forma más profunda en un curso de ciencias (especialmente a partir de una perspectiva cognitiva-constructivista), al no indicar aspectos relativos al proceso de aprendizaje. Se puede decir esto con alguna seguridad, pues, como el propio Bloom ya admite (1956), el uso de la taxonomía no proporciona diferenciadores claros y completos entre un mismo comportamiento esperado que haya sido alcanzado por distintos alumnos de maneras diferentes.

Y por añadidura, si utilizamos pruebas escritas basándonos solamente en la taxonomía, poco podremos decir al respecto de alumnos que no lleguen a mostrar determi- 
nados comportamientos esperados. Obsérvese, por ejemplo, cuando un alumno no demuestra el comportamiento de aplicación. ¿Qué podemos decir de esta no-manifestación de manera que podamos ofrecer un instrumento más palpable al profesor y que le permita trabajar mejor que con aquél de Bloom (1956,1971), al sugerir que los fracasos pueden estar relacionados, en líneas generales, con una comprensión, selección o uso inadecuado de una misteriosa «abstracción con respecto al asunto?».

En este trabajo pretendo exponer algunas ideas y un modelo de prueba escrita que intenta, de forma preliminar pero con resultados positivos, suministrar algunos elementos que se refieren al proceso de enseñanzaaprendizaje en sí, de una manera más significativa que lo que podría ser hecho con la sola utilización de la taxonomía de Bloom. El uso de estos elementos debe, por lo tanto, ser capaz de retroalimentar las actividades de los docentes y de los estudiantes a través de una perspectiva actualizada de enseñanza de las ciencias: aquélla del aprendizaje de las ciencias como investigación (Gil 1993) -punto que será objeto de discusión al final del artículo.

\section{ALGUNAS LIMITACIONES OUE DEBEN SER CONSIDERADAS EN EL USO DE LA TAXONOMIA DE BLOOM}

\section{La dependencia de las experiencias anteriores}

En lo que se refiere al dominio cognitivo, la taxonomía de Bloom es útil para realizar una diferenciación de los distintos objetivos educativos en las pruebas o ejercicios. Sin embargo, debemos tener en consideración algunas de sus limitaciones.

Inicialmente, como ya manifestara Bloom (1956), «una de las principales dificultades para categorizar las cuestiones de una prueba consiste en saber que es necesario, en todos los casos, conocer o considerar las experiencias educativas anteriores de los alumnos sometidos a la prueba». O sea, si el alumno se encuentra frente a una situación nueva, su solución puede requerir comportamientos mucho más complejos que en el caso de que la misma situación le sea presentada y que no fuese una novedad. En este caso, la solución de un problema podría, inclusive, ser alcanzada a través de procesos de reconocimiento y memoria.

Para ilustrar este argumento veamos el problema $\mathrm{A}$, identificado por Bloom (1971) como una cuestión que puede requerit el comportamiento de asumir con anticipación, a partir de una serie de criterios y relaciones, qué suposiciones pueden ser implícitas, solicitadas o necesarias; o sea, uno de los típos de comportamiento de análisis. En el caso de que un profesor haya discutido ese mismo ejemplo en sus clases, sólo por reconocimiento y memoria, el alumno podría responder correctamente a esa pregunta o ese tema, lo que la remititía en la taxonomía a ser catalogada como una cuestión del comportamiento del conocimiento.
Problema A: Déjeme explicar cómo usted puede establecer si una cosa o evento es causa de otra. Hablamos de dos cosas o eventos « $x » e$ «y». Si en cualquier momento usted comienza a observar que $X$ ocurre y despues de esto nota que $Y$ siempre sigue a $X$ y que nunca ocurre $Y$ excepto después de $X$, entonces usted puede decir con seguridad que $X$ es la causa de $Y$. Empecé a realizar estas observaciones y reparé que cuando el sol se ocuitaba siempre era seguido más tarde por el surgimiento del sol y que el sol nunca nace sin haberse ocultado antes.

\section{Comentarios:}

A. La conclusión se realiza logicamente.

B. La segunda parte de las explicaciones usa injustamente los argumentos de la primera.

C. Una suposición importante fue omitida en los argumentos. D. La conclusión es absurda, por consiguiente no puede tener lógica.

E. La declaración de causa es falaz.

\section{Feedback limitado y la cuestión de las capacidades}

Como ya comentamos anteriormente, profesores preocupados con el proceso de aprendizaje en sí, limitan su feedback al utilizar solamente la taxonomía. Esto sucede porque ésta no consigue mostrar qué provocó que un alumno manifestara o no un determinado comportamiento y por eso no es capaz de señalar qué hace falta o qué debería ser más trabajado por el profesor para que, si ése fuese el objetivo del curso, ese comportamiento determinado sea alcanzado por los alumnos que aún no lo manifiesten. Para entender esto más claramente y percibir las habilidades que están subyacentes a las categorías taxonómicas de Bloom, veamos los problemas B y C. Al analizar el asunto B considerado por Bloom (1956) como referente al comportamiento do aplicación, vemos que este asunto no sólo encierra comportamientos diferentes (como ellos reconocen) sino que también exige habilidades diferentes (que no indicaron) como aquéllas que son necesarias para resolver el problema $C$, también considerado un problema para comprobar o verificar comportamientos de aplicación (Bloom et al., 1971). (Lea la observación después de leer los asuntos B y C.)

En el problema B, el alumno requiere, evidentemente, una gran habilidad para poder elaborar un texto claro (expresión escrita) y, al "fundamentar» su respuesta, necesita tener bien claros los criterios (percibir que existen diferentes elementos particulares dentro del fenómeno) que va a utilizar.

Tener criterios establecidos es importante para solucionar el problema $C$ (diferenciar y distinguir el papel de "g" y de la resistencia del aire en la caída libre de los cuerpos). Sin embargo, la respuesta dada puede ser correcta si el alumno tiene la percepción de un determinado aspecto más específico (aquél que se refiere solamente a la fuerza de gravedad y a la resistencia del aire en la caída libre de los cuerpos). Ya en el problema B, para que haya una respuesta completa y no parcial, el alumno debe demostrar que tiene una percepción más global, de los diversos aspectos que están en interacción en esa situación y que no fueron especificados en el problema. 
Problema B: Juan preparó un acuario de la siguiente manera: Limpió cuidadosamente, con una solución salina, un recipiente de vidrio con capacidad para 10 galones*. Cubrió el fondo con algunas pulgadas de arena fina lavada y plantó algunas algas (elodes) que recogió en un lago; después llenó el acuario con agua. Una semana después colocó diez peces dorados pequeños y tres caracoles. Dejó el acuario en una esquina. Un mes después descubrió que el agua no estaba fétida y que las plantas y los animales estaban en perfectas condiciones. Sin mover el acuario, Juan lo cerró con una tapa de vidrio.

¿Cuál sería su previsión-si puede ser hecha-sobre las condiciontes del acuario después de que transcurran varios meses? Si usted cree que una previsión definitiva puede ser realizada, hágala y fundaméntela. Si usted se considera incapaz de realizar tuna previsión por cualquier razón, indique las causas.

${ }^{*}$ Galón: medida de capacidad equivalente a 3,785 litros.

Problema C: En el uso de la ecuación $\mathrm{s}=1 / 2 \mathrm{gt}^{2}$ para calcular el tiempo que demora un determinado cuerpo al caer desde unta aitura h del suelo, ¿cuál de los factores seíalados a continuación produciría un mayor error?

a) factor 1 (variación de la gravedad);

b) factor 2 (variación de la resistencia del aire);

c) factor 1 para alturas superiores a un cierto valor, factor 2 para alturas menores:

d) factor 2 para alturas superiores a un cierto valor, factor 1 para alturas menores.

Observación: Según Bloom, el problema B requiere que el alumno seleccione principios y extrapole datos de la situación; el problema $C$ requiere del alumno el comportamiento de especificar límites, condiciones especiales bajo las cuales la generalización o el principio son verdaderos. Estos dos comportamientos son considerados en la taxonomía como tipos de comportamientos de aplicación.

Por lo tanto, al encontrarse con cualquiet situación, el alumno y también el profesor o el científico, necesitan tener una serie de habilidades para poder manejarla. Como esas habilidades son utilizadas junto con un determinado conocimiento de los hechos, conceptos y principios que se refieren a una situación específica, evitaremos, de aquí en adelante, usat el término habilidad. Para designar esta asociación de habilidad + un contenido determinado usaré un término que Bloom (1956) ya definió con ese mismo sentido: capacidad.

De esta forma, para manifestar cualquiera de los comportamientos estipulados en la taxonomía, son necesarias varias capacidades. Algunas de éstas, siendo posiblemente subyacentes a las categorías taxonómicas ya establecidas, no fueron siquiera detalladas por Bloom. Esto probablemente debe haber ocurrido porque los estudios realizados para la elaboración de la taxonomía enfocaban los comportanientos considerados como resultado del proceso educativo, y los autores de la taxonomía se limitaban a citar brevemente el tema de las capacidades sin profundizarlo.

Si aplicáramos pruebas y las corrigiéramos ignorando la idea de capacidades subyacentes a las categorías taxonómicas o si las hiciéramos basándonos solamente en la taxonomía de Bloom, tenderíamos más a «diagnosticar» cuáles de los objetivos de comportamiento taxonómicos fueron verificados y cuáles no, que a incorporar medidas y reflexiones pedagógicas más relacionadas con el proceso de aprendizaje.

Muchos profesores, al no considerar la cuestión de las capacidades, pueden inclusive juzgar de forma errónea que, si un alumno no manifiesta comportamientos considerados como de aplicación, por ejemplo, debe ser sometido a ejercicios y actividades de aplicación (o de cualquier otro objetivo que el alumno no haya manifestado) para que en un futuro próximo el estudiante demuestre esos comportamientos.

Más adelante explicaré, daré ejemplos e indicaré cómo cada uno de los cinco tipos de preguntas diferentes que establecí dentro del modelo de prueba escrita que estoy utilizando puede ofrecer indicios sobre diferentes capacidades de los alumnos relacionadas con la construcción de conocimientos científicos.

Debo, evidentemente, hablar de «modelo» de prueba, pues, como ya matizaron Rodríguez y sus colaboradores (1992), una determinada técnica de evaluación no constituye un método de investigación, puesto que «los datos obtenidos con aquélla nunca se explican por sí mismos, sino que su interpretación está condicionada por los supuestos teóricos del investigador". Siendo así, se hace necesario explicar el modelo teórico de interacción entre capacidades que está sobreentendido en el modelo de prueba escrita que vengo utilizando.

\section{MODELO TEÓRICO DE INTERACCIÓN ENTRE LAS CAPACIDADES}

\section{Las capacidades primitivas y derivadas}

El modelo teórico de interacción entre capacidades aquí propuesto admite que, en el proceso de construcción de un conocimiento dado, son necesarias diferentes capacidades. Estas capacidades no son equivalentes, o sea, algunas son más básicas (primitivas) y otras son derivadas de éstas.

Las capacidades más básicas (bases para la construcción de un conocimiento) que hasta el momento hemos conseguido diferenciar, se han agrupado dentro de un conjunto denominado nivel de las capacidades subyacentes (Fig. 1), dividido en su vez en dos subconjuntos: uno con las capacidades de la memoria, expresión y percepción particular; y ofro con las capacidades de criterio (o de percepción aguda), crítica y percepción global.

El primero de estos subconjuntos puede ser considerado como aquél con las capacidades primitivas dentro del nivel, el segundo engloba las capacidades derivadas dentro de ese mismo nivel. Esto es, a partir de la interacción entre las capacidades de memoria, expresión y percepción particular (consideradas subyacentes y pri- 
marias), derivan las capacidades de criterio, crítica y percepción global (consideradas subyacentes y derivadas).

Las capacidades subyacentes -así como aquéllas de los demás niveles- que estén en desarrollo contribuyen en el surgimiento y desarrollo de varios "procesos mentales" relacionados con el aprendizaje. Tomemos como ejem plo la capacidad de expresión, específicamente, la capacidad de expresión escrita, que es el tipo de expresión que me limito abordar en este artículo.

Por capacidad de expresión escrita entiendo el uso de los recursos materiales de la lengua escrita. Estos recursos pueden ser la construcción de tablas, gráficos, cuadros, redacción de textos, etc. El uso de esos recursos, particularmente la redacción de textos, es un importante elemento que, al ser desarrollado, se asocia no sólo al aumento de consciencia, sino al desarrollo de procesos mentales avanzados, como el propio pensamiento conceptual. El desarrollo de esta capaciodad de expresión también es asociada a la traducción compleja que el alumno va siendo capaz de realizar, con el paso del tiempo, de sus pensamientos y conceptos que están en el plano abstracto, al plano concreto.

Sin embargo, esos «procesos mentales» realacionados con el aprendizaje (como, por ejemplo, tomar consciencia, desarrollar el pensamiento conceptual junto con la creación de significaciones, la traducción de lo abstracto a lo concreto), no ocurrirán solamente con el desarrollo de la capacidad de expresión escrita. Siendo procesos muy elaborados y refinados, en ese modelo se sobreentiende que sus surgimientos están vinculados a las capacidades de conocimiento y comprensión amplios, las que se encuentran en el nivel de las capacidades avanzadas finales, o sea, aquéllas que se derivan de todas las demás (Fig. 1).

En otras palabras, las capacidades definidas en la figura 1 pueden ser consideradas como representantes directas de «funciones mentales elementales». $Y$, recordando las palabras de Vygotsky (1934 $)$, «un concepto se forma no por la interacción de asociaciones, sino mediante una operacion intelectual en que todas las funciones mentales elementales participan en una combinación especifíca. Esa operación es dirigida por el uso de las palabras como medio de centralizar activamente la atención, abstraer determinados trazos, sintetizarlos y simbolizarlos por medio de un signo".

Siendo así, tanto el surgimiento de esos procesos como el de las capacidades avanzadas finales es vinculado al desarrollo de las capacidades consideradas en los demás niveles, las cuales se relacionan a su vez con manifestaciones típicas que empiezan a aparecer en cada nivel y que pueden ser percibidas por el profesor.

\section{Niveles, capacidades y manifestaciones}

En este modelo teórico de interacción entre capacidades hemos definido como nivel un conjunto dado de capaci- dades que se asocian a un conjunto dado de manifestaciones externas que un alumno puede demostrar. De esa forma el nivel de las capacidades subyacentes se caracteriza por estar vinculado a manifestaciones como relaciones, discusión y argumentación preliminares y simples de los contenidos (o sea que no revelan mucho análisis, síntesis y juicio, sino sólo las «percepciones iniciales»). Alumnos que estén desartollando las capacidades de este nivel comienzan también a mejorar su discurso (oral y escrito), el cual, es como si fuese un mosaico de partes diversas, con algunas más claras y otras confusas.

\section{El nivel de las capacidades iniciales}

En ese modelo se asume que, al pasar del nivel de las capacidades subyacentes al nivel de las capacidades avanzadas iniciales (Fig. 1), los alumnos comienzan a elaborar definiciones y procedimientos, a describir y a explicar contenidos con una cierta claridad, que va en aumento. Asociada a esas manifestaciones, típicas del nivel de las capacidades iniciales, está el desarrollo de las capacidades primarias dentro de ese nivel (la de conocimiento y comprensión en sentido estricto) y derivadas (la de análisis, evaluación y síntesis en sentido estricto). Hemos preferido, en este caso, agrupar las capacidades primarias y derivadas en el nivel de las capacidades iniciales en lugar de separarlas en niveles distintos, con la finalidad de centralizar la atención en las manifestaciones que consideramos que son comunes a ellas y que resultan típicas del nivel de las capacidades iniciales.

De esta forma, la capacidad de conocimiento dentro de este nivel, y por lo tanto en un sentido estricto, designa las habilidades para el conocimiento de un determinado contenido en un nivel, que es un poco mayor que la capacidad de memoria. Al final aquí estamos tratando de una capacidad menos subyacente, que incluye no sólo la capacidad de percepción particular, un desarrollo de la capacidad de expresión, sino también otras capacidades subyacentes que discutiré más adelante.

Además, es en el desarrollo de la capacidad subyacente de expresión donde está la diferencia entre estas dos capacidades tomadas en sentido estricto -la del conocimiento y la de la comprensión. Así, la capacidad de comprensión en el nivel inicial, en comparación con la capacidad de conocimiento en ese mismo nivel, envuelve un mayor dominio de la capacidad de expresión. Un fruto directo de eso, y que por to tanto nos indica la existencia de la capacidad de comprensión estricta, es la manifestación de la traducción simple, o sea, de un contenido para gráficos, tablas y textos o viceversa.

Ya entre las capacidades derivadas en ese nivel, la capacidad de síntesis, en un sentido más estricto, se tefiere al dominio inicial de operaciones para combinar determinados elementos de un contenido dándoles nuevas configuraciones. La capacidad de análisis, también en sentido estricto, se refiere a su vez al dominio inicial de operaciones para fragmentar un determinado asunto 
en partes (y no simplemente de percibir la existencia de aspectos particulares en un fenómeno, lo que caracteriza la capacidad subyacente de criterio). Y la capacidad de evaluación (que concierne a las habilidades relacionadas con un juicio y no simplemente con una posición del individuo), que aquí también es definida en un sentido estricto, se refiere a la manifestación de una opinión más egocéntrica basada en criterios y fundamentada en los juicios personales que envuelven esos contenidos.

En este modelo asumo que la presencia de todas las capacidades en los niveles inicial (las que ya hemos definido) y subyacentes (las que explicaremos más profundamente) posibilita el surgimiento y desarrollo de capacidades más avanzadas que explico a continuación.

\section{Las capacidades avanzadas y el surgimiento de las hipótesis}

Arbitrariamente dividimos las capacidades avanzadas en tres niveles, el inicial, el intermedio y el final, ya que consideramos que cada uno de estos niveles presenta manifestaciones características, que bien diferenciadas son de gran utilidad para el trabajo del profesor. Ast, en el nivel de las capacidades avanzadas iniciales se encuentran las capacidades de conocimiento y compren. sión, no en sentido estricto pero tampoco en un sentido amplio, lo que sólo ocurre en el nivel de las capacidades avanzadas finales.

Las capacidades de conocimiento y comprensión en el nivel avanzado inicial están relacionadas, por lo tanto, con una mayor vinculación y traducción interpretativa que los alumnos consiguen hacer de los hechos. Llamo traducción interpretativa a la manifestación de los alumnos que no se limita a traducir contenidos en gráficos, tablas y textos (o lo contrario) de una forma impersonal. En la traducción interpretativa sobreentiendo que en el pro. ducto final se encuentra claramente la traducción del hecho (lo que denominé de traducción simple), así como también están presentes, de manera notable, comentarios personales del traductor sobre los hechos.

La diferencia entre el lado personal y el lado factual además de estar presente en la distinción de la capacidad de criterio y crítica (las cuales discutiremos más adelante), de traducción simple y traducción interpretativa, también está presente en la propia diferencia que realizamos entre las capacidades de conocimiento y com*

Figura 1

Diagrama esquematizado de las capacidades, de los niveles de capacidades y de las manifestaciones de cada nivel que fueron discutidas en cl artículo.

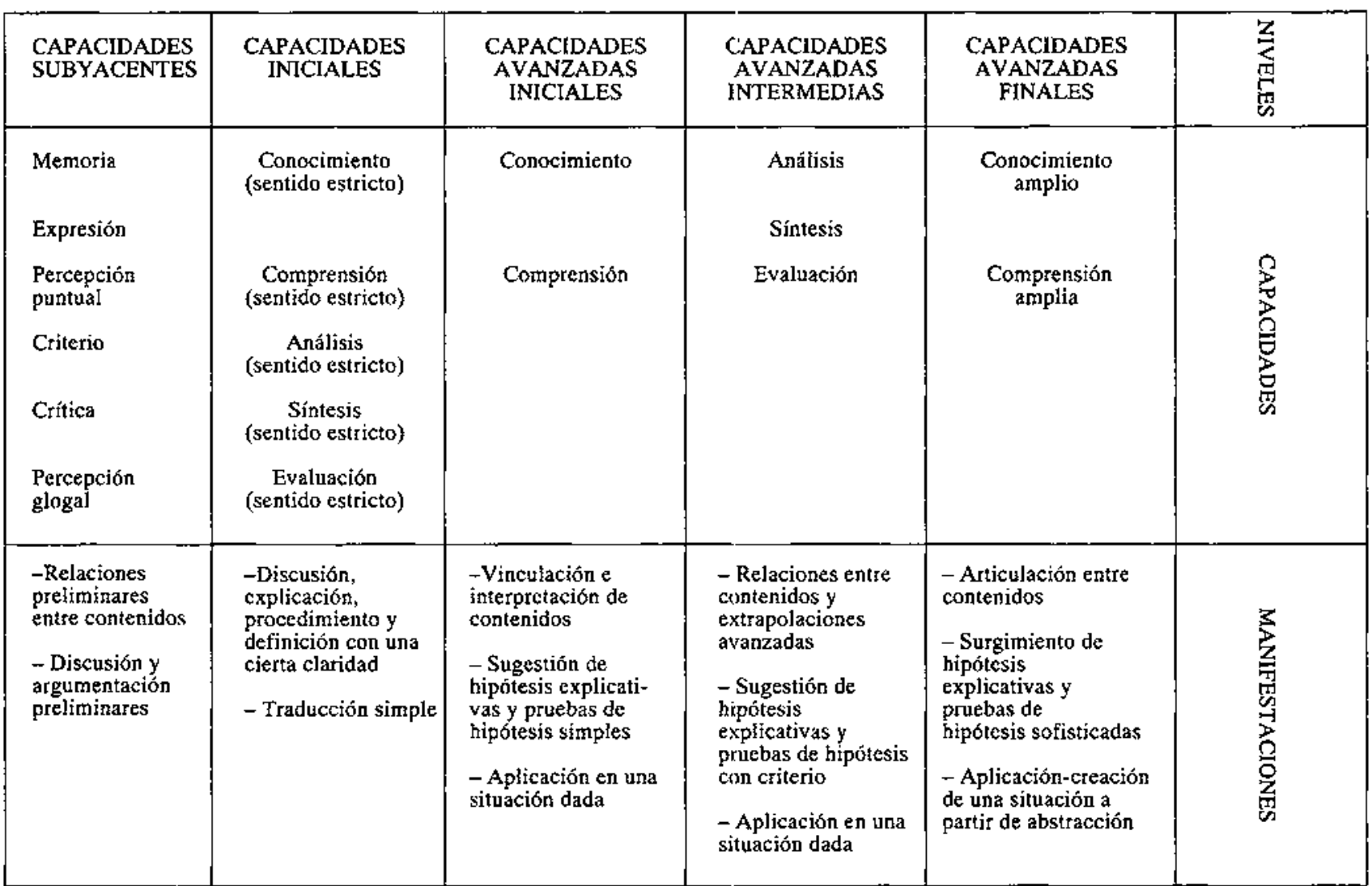


prensión no consideradas en sentido estricto. En ese caso, la primera está más ligada a los hechos y la segunda a "puntos de vista" basados en los hechos.

Los alumnos que tienen un conocimiento y comprensión situados dentro del nivel de las capacidades avanzadas iniciales demuestran no solamente una interpretación y vinculación de contenidos más acentuada que aquéllos con el conocimiento y comprensión restringido, sino que también comienzan a sugerir algunas hipótesis explicativas y pruebas de hipótesis simples.

Esas hipótesis explicativas y las pruebas de hipótesis que están vinculadas a ellas van sofisticándose y se enriquecen por las relaciones entre contenidos y extrapolaciones a medida que se desenvuelven las capacidades de análisis, síntesis y evaluación dentro del nivel de las capacidades avanzadas intermedias. En ese nivel, el análisis, se demuestra con mucho más criterio que, en el nivel inicial, la sintesis puede ocurrir teniendo como base no solamente los hechos, sino también derivaciones abstractas, y la evaluación (o juicio) es más fundamentada que egocéntrica.

Alumnos que dominen capacidades en esos niveles consiguen alcanzar el objetivo comportamental de Bloom sobre aplicación. Siendo así, vale la pena destacar que, en ese modelo de interacción entre capacidades, son presentadas como capacidades reales solamente aquellas habilidades que se refieren a un contenido determinado dado que juzgue ser las fundamentales en el proceso de construcción del conocimiento. Consecuentemente, la aplicación aquí no debe ser confundida con una capacidad real, a fin de cuentas se constituiría solamente en una de las manifestaciones referente a las capacidades avanzadas presentadas.

Esta manifestación típica del nivel avanzado, la de la aplicación, también puede sofisticarse, asociándose no sólo a la aplicación de un conocimiento en una situación dada, sino también a la propia creación de una situación concreta a partir de abstracciones. Ese último tipo de manifestación de aplicación, a su vez, se relaciona fuertemente con alumnos que dominen la capacidad de conocimiento y comprensión en un sentido amplio.

Alumnos con capacidad de conocimiento y comprensión amplios tambiên pueden manifestar una articulación entre contenidos -que no podía ser constatada-, así como su elaboración de hipótesis y pruebas de hipótesis pasan a ser sofisticadas. Esos alumnos también pueden demostrar los frutos del dominio de las capacidades aquí presentadas y que, por lo tanto, se encuentran íntimamente asociadas al conocimiento y comprensión amplios: la toma de consciencia, el desarrollo del pensamiento conceptual acompañado de la creación de conceptos y la traducción de lo abstracto a lo concreto.

Como este esquema de interacción entre capacidades no es lineal, se hace fácil entender que esos mismos alumnos, en un futuro próximo, puedan comenzar a presentar manifestaciones referentes a los otros niveles de capacidades, inclusive aquéllas que no son características de los niveles avanzados, en la medida en que el conocimiento y comprensión amplios que presentan sean remodelado en el futuro, en un proceso teóricamente interminable de construcción y reconstrucción del conocimiento.

\section{EL MODELO DE PRUEBA ESCRITA}

\section{Líneas generales del modelo}

El modelo de prueba escrita que adoptamos se basa en el modelo teórico que hemos expuesto, particularmente en el hecho de diferenciar capacidades reales de manifestaciones externas y de identificar cuáles de las úitimas se refieren a un nivel dado de capacidades. Por lo tanto, al hacer o clasificar preguntas dentro de una prueba, intentamos inicialmente tener bien clara la tabla de respuestas con las manifestaciones exigidas en las respuestas de los alumnos para que éstas sean consideradas correctas. A partir de aquí se pretende identificar cuáles son las capacidades que se encuentran marcadamente contempladas en los diferentes tipos de pregunta.

Siguiendo este tipo de procedimiento hemos llegado, hasta el momento, a establecer la existencia de cinco tipos de preguntas caracterizadas pot demandar capacidades en los diferentes niveles además de ser capaces de indicar, evidentemente en las respuestas que los alumnos dan, un diferente dominio de las capacidades subyacentes (Fig. 2). Exactamente por ser definidas dentro de esas características, un modelo de prueba con esos tipos de preguntas puede ser un elemento de gran utilidad en la retroalimentación de la actividad docente y de los estudiantes.

\section{TIPOS DE PREGUNTAS Y CAPACIDADES RELACIONADAS}

\section{Preguntas ultraobjetivas}

Las preguntas ultraobjetivas se caracterizan por requerir de los alumnos, predominantemente, el uso de la capacidad de memoria. Por eso entendemos el uso de operaciones mentales que envuelven recuerdos o ellamados", recapitulación en el plano abstracto y evocación de un contenido dado. Alumnos que demuestren tener un grado de desarrollo alto o medio tendiendo a alto en esa capacidad acostumbran a acertar en ese tipo de pregunta.

Alumnos que no demuestren (por cualquier motivo) o que tengan un desarrollo bajo en esa capacidad erran ese tipo de pregunta. Vale la pena recordar que estos últimos pueden tener una buena habilidad de memoria y demostrarlo en lo concerniente a varios temas. Sin embargo, como no demuestran esta habilidad en lo que se refiere a este tema específico, no se detecta, en este caso, la capacidad de memoria. 
Los altumnos que tengan un grado de desarrollo medio de la capacidad de memoria podrán responder correctamente a las preguntas del tipo ultraobjetivas si poseen otras capacidades subyacentes que, en este caso, le serán de utilidad: la de expresión y la de percepción particular.

La capacidad de percepción, de forma general, significa simplemente "percibir" un contenido, o sea, tener una leve claridad respecto a él en el plano consciente (lo que no está sobreentendido en la capaciđad de memoria). Esa percepción no tiene, por lo tanto, la misma connotación, y ni es tan superyacente como la comprensión o el conocimiento de un contenido dado.

Como en los preguntas ultraobjetivas, esa percepción se refiere a aspectos más «circunscritos» del conocimiento, se denomina percepción particular, oponiéndose a la capacidad de percepción global. Esta última, como su nombre expresa, no se refiere a aspectos circunscritos del conocimiento. Es decir, la capacidad de percepción global incluye una percepción mayor de diferentes aspectos del conocimiento que están articulándose, siendo extremamente necesaria en otros tipos de preguntas que veremos más adelante.

He observado que la articulación más especifica entre las capacidades de expresión escrita y percepción particular, dentro de las preguntas ultraobjetivas, es de gran ayuda para que un alumno con un grado de desarrollo medio de la capacidad de memoria consiga respuestas correctas. Pero como ya establecimos anteriormente, en ese tipo de pregunta, la memoria es la capacidad dominante. Esto significa que constatamos cómo alumnos que poseen un grado de desarrollo alto de cualquiera otra de las capacidades que estoy discutiendo en este artículo, en este tipo de pregunta, pueden sentir más facilidad o no, pero de cualquier forma tendrán el mismo resultado que los alumnos que tienen exclusivamente, o casi, una capacidad de memoria alta. Ese resultado llevaría a una respuesta correcta.

Un esquema que resume los grados de desarrollo de las capacidades subyacentes que pueden ser reveladas en los diferentes tipos de pregunta se encuentra en la figura 2. En el final del artículo también presentamos algunos ejemplos de cuestiones que ilustran lo que sería una pregunta ultraobjetiva. Analizando estas cuestiones podemos constatar que, como la categorización de Bloom, la decisión de que una pregunta dada sea o no ultraobjetiva se define en el contexto del aula, puesto que preguntas que en principio envuelvan una serie de capacidades pueden, dependiendo del trabajo del profesor, ser respondidas con el uso predominante de la capacidad de memoria. (Véanse como ejemplo las preguntas 27 y 17 ).

No obstante, podremos percibir, en la secuencia del artículo, que en la categorización de algunas preguntas, principalmente aquellas que incluyen aspectos personales en las respuestas, aunque el trabajo realizado por el profesor las obligue a utilizar en las respuestas la capacidad de memoria, aún continúan siendo necesarias otras capacidades para garantizar una respuesta correcta (Véanse como ejemplo las preguntas 14 y 21 ). Por otra parte, lo inverso también puede ocurrir; esto es, algunas preguntas considerados cono ultraobjetivas jamás podrían ser reconocidas como preguntas que incluyan otras capacidades además de la memoria (ejemplo, pregunta 6).

\section{Preguntas objetivas}

Las preguntas que Ilamamos objetivas necesitan, para ser respondidas correctamente, las mismas tres capacidades que son requisitos en las preguntas ultraobjetivas. Sin embargo el peso que tienen la capacidad de expresión escrita y la percepción particular es mayor que el de la memoria (Fig. 2). Tomando en consideración el modelo teórico de interacción entre capacidades podemos afirmar que los alumnos que responden correctamente a esas preguntas ya presentan, por lo memos, las capacidades de conocimiento y comprensión extrictos, y pueden demostrar claramente las manifestaciones referentes al nivel de las capacidades iniciales.

Por lo tanto, simplificadamente podríamos decir que, mientras las preguntas ultraobjetivas exigen que el alumno «manifieste» un contenido a través de la capacidad de memoria, las preguntas objetivas exigen del estudiante ura definición, descripción, realización o explicación de ese contenido que serían insuficientes con el exclusivo uso de la capacidad de memoria.

Basándonos en la figura 2 y en las preguntas que se encuentran en los anexos podemos notar que alumnos con capacidad de expresión escrita y percepción particular deficientes consiguen responder correctamente a una pregunta ultraobjetiva. Sin embargo, el alumno necesita tener un grado de desarrollo medio, por lo menos, en esas dos capacidades para responder correctamente una pregunta objetiva.

También en las preguntas objetivas, al contrario de las ultraobjetivas, poseer un grado de desarrollo alto de la cixpacidad de expresión escríta y percepción particular puede ser importante para garantizar una respuesta correcta. Esto no ocurre en lo que se refiere a la capacidad de memoria, pues ésta, aun siendo fuerte en el alumno que responda a ese tipo de pregunta, no es un elemento preponderante para garantizar aisladamente que la respuesta esté correcta.

Diferentes estrategias pueden ser utilizadas para elaborar una pregunta objetiva. Podemos exigir un aspecto descriptivo, uno explicativo, elaboración de procedimientos y definiciones o pequeños ensayos que incluyan todos estos aspectos simultáneamente. AI final de este artículo encontraremos ejemplos de preguntas objetivas que, dependiendo del enfoque de la pregunta, pueden, por lo tanto, ser clasificadas como preguntas objetivas de descripción (pregunta 7), definición (pregunta 9 y parte de la 10), procedimiento (pregunta 8) y explicación (preguntas 11, 12 y 13). A pesar de que esas mismas estrategias pueden ser utilizadas en la elaboración de otros tipos de preguntas, preferimos, hasta el momento, identificarias solamente como una clasificación para 
Figura 2

Cuadro sinóptico de los grados de desarrollo en que son requeridas las diferentes capacidades subyacentes para que haya una respuesta correcta a un determinado tipo de pregunta.

\begin{tabular}{|l|c|c|c|c|c|}
\hline $\begin{array}{l}\text { Capacidades } \\
\text { subyacentes }\end{array}$ & Objetiva & $\begin{array}{l}\text { Objetiva de } \\
\text { discusión } \\
\text { Objetiva }\end{array}$ & $\begin{array}{l}\text { Discusión y pregunta } \\
\text { argumentación }\end{array}$ & $\begin{array}{l}\text { Asociación de } \\
\text { conocimientos }\end{array}$ \\
\hline Memoria & 4 & 4 & 4 & 4 & 4 \\
\hline Expresión & 1 & 3 & 3 & 4 & 4 \\
\hline Percepción puntuat & 1 & 3 & 4 & 3 & 4 \\
\hline Criterio & & & 2 & 3 & 4 \\
\hline Crítica & & & & 2 & 3 \\
\hline Percepción global & & & & 4 \\
\hline
\end{tabular}

La capacidad debe estar presente en un grado de desarrollo:

1. Por lo menos bajo, pero igual en grado alto; esto no favorece una respuesta correcta.

2. Por lo menos bajo.

3. Por to menos medio.

4. Preferentemente medio con tendencia a fuerte.

diferenciar preguntas del tipo objetivo, debido a que estas preguntas están dirigidas hacia Ia verificación de manifestaciones referentes al nivel de las capacidades iniciales.

Somos conscientes de que to que nosetros hemos denominado preguntas ultraobjetivas son para muchos docentes simplemente cuestiones objetivas. $Y$ que lo que en nuestro modelo son preguntas objetivas, se consideran en general como preguntas conceptuales de desarrollo corto.

\section{Preguntas objetivas de discusión}

Las preguntas objetivas de discusión to son presentadas junto con los tipos de preguntas objetivas establecidas anteriormente porque requieren, además de las capacidades subyacentes ya explicadas, una nueva capacidad que no es necesaria para garantizar una respuesta correcta en una pregunta objetiva. Esa capacidad se liama criterio.

Por capacidad de criterio, entendemos la habilidad de percibir la existencia de aspectos particulares dentro de un determinado fenómeno más amplio. Esta capacidad, por lo tanto, no puede ser confundida con la capacidad de análisis, evaluación o síntesis, las cuales son más sofịsticadas y derivadas en gran parte de la capacidad de criterio. Además de esto, la capacidad de criterio no puede ser confundida con la capacidad de percepción particular, pues ésta sobreentiende más que una leve claridad, incluye la percepción ligeramente aguda de un contenido.
De esa forma es posible admitir que la capacidad de criterio es una capacidad subyacente que presenta innovaciones con relación a las demás capacidades subyacentes que hemos visto hasta ahora. Podemos inclusive decir esto con cierta seguridad debido al hecho de que en las pruebas, donde los alumnos obtienen respuestas correctas solamente en las preguntas objetivas y ultraobjetivas, se verifica que muchas de las respuestas a las preguntas que requieren la capacidad de criterio están parcialmente correctas o equivocadas.

En el final del artículo se encuentran algunos ejemplos de preguntas objetivas de discusión. Para responderlas, el alumno debe poseer una capacidad de criterio baja (con preferencia tendiendo a media) y una percepción particular superior a la media. Sin embargo constatamos que, en términos generales, los alumnos que obtieneri una respuesta correcta en ese tipo de pregunta son aquéllos que demuestran, en los demás tópicos de una prueba, una fuerte capacidad de percepción particular.

Como las preguntas que presentamos no son solamente de escoger la mejor respuesta y exigen que el alumno escriba, en las preguntas objetivas de discusión (así comoen las objetivas), es necesario que el alumno tenga un grado de desarrollo por lo menos medio en la capacidad de expresión escrita. Alumnos con capacidad de expresión escrita baja no consiguen responder correctamente las preguntas objetivas de discusión ni preguntas objetivas que no sean aquéllas de escoger la mejor respuesta.

De igual forma que en las preguntas objetivas, en las preguntas objetivas de discusión y en las demás que serán expuestas, los alumnos deben poseer un grado de 
desarrollo entre medio y alto en lo que se tefiere a la capacidad de memoria. Alumnos con baja capacidad de memoria, en términos generales, en toda pregunta de ese modelo de prueba no tienen posibilidades de obtener ninguna respuesta totalmente correcta.

Vale la pena resaltar que, asociada a la conquista de los grados de desarrollo medio y alto de las diferentes capacidades subyacentes (incluyendo la de memoria), parece que se realiza simultáneamente en los alumnos el desarrollo de capacidades más superyacentes. Por lo tanto, debemos tener claro que en el modelo teórico de interacción entre las capacidades, ya expuesto, existe un proceso extremamente dinámico e interminable de interrelación entre capacidades en diferentes niveles, lo que fomenta el desarrollo de capacidades dentro de un determinado nivel, el surgimiento de capacidades más derivadas y así una constante remodelación del conocimiento y de la comprensión del contenido.

\section{Las preguntas de discusión y argumentación}

Para ser respondidas correctamente las preguntas de discusión y argumentación requieren un grado de desarollo superior al medio en las capacidades de expresión escrita, percepción particular y memoria, así como exigen del alumno un nivel, por lo menos, medio de la capacidad de criterio.

Las capacidades de percepción global, aunque ayudan en las respuestas a los demás tipos de preguntas, principalmente las objetivas de discusion, no constituían hasta ahora un elemento obligatorio para garantizar una respuesta correcta. A fin de cuentas, existen alumnos que responden correctamente las preguntas ultraobjetivas, objetivas y objetivas de discusión y erran en las preguntas de discusión, argumentación y asociación de conocimiento, las que exigen nítidamente un cierto nivel de capacidad de percepción global.

Particularmente en las preguntas de discusión y argumentación se observa que la presencia de la capacidad de percepción global, aunque en bajo grado, es constante en Ios aíumnos que obtienen respuestas correctas. Además de eso, en este tipo de preguntas es necesaria una nueva capacidad subyacente que aún no presentamos: la capacidad de crítica, por lo menos en un nivel medio de desarrollo.

Por capacidad crítica entendemos las habilidades vinculadas a la posición personal de un individuo (en las cuales no se realiza un juicio) con relación a las evidencias que están detrás de un fenómeno dado. Las capacidades de criterio y de crítica caminan próximas; sin embargo, podemos distinguirlas porque la capacidad de criterio se refiere más a un fenómeno dado, mientras que la capacidad de crítica se refiere a una posición personal respecto a un fenomeno.

He observado que algunos alumnos, además de comenzar a establecer relaciones (pese a que no son sofisticadas) con mayor frecuencia que antes respecto a un contenido đado, también empiezan a realizar discusiones y a presentar argumentos defendiendo sus opiniones. Estos alumnos, además de mostrar manifestaciones referentes a los diferentes niveles de capacidades (como explicación, descripción, sugerencias de hipótesis, etc.), obtienen en las pruebas muchas más respuestas correctas que antes en las preguntas objetivas de discusión y principalmente en las de discusión y argumentación. Creo que eso demuestra que esas preguntas nos dan indicios de la transición que está ocurriendo en los alumnos, del nivel de las capacidades iniciales hacia los niveles de capacidades avanzadas, además de que se constituyen en una evidencia real de la dinámica de interacción -y nos sugieren la existencia de un proceso interminable-entre las capacidades en la construcción del conocimiento.

\section{Las preguntas de asociación de conocimientos y de aplicación de las capacidades avanzadas}

Las preguntas de asociación de conocimientos son respondidas correctamente por alumnos que demuestren tener capacidad de criterio, crítica y percepción global en un grado superior al de los alumnos que obtienen respuestas correctas en las preguntas de discusión y argumentación y se equivocan en las de asociación de conocimiento. Son, por lo tanto, necesarios (Fig. 2) un grado de desarrollo, por lo menos medio de la capacidad de percepción global, y un grado de desarrollo superior al medio en la capacidad de criterio y crítica (además de esos mismos requisitos en lo que se refiere a las otras capacidades) para obtener respuestas correctas en ese tipo de preguntas.

Al comparar las correcciones de las preguntas de asociación de conocimiento y de discusión y argumentación, se observa que diferentes alumnos que obtuvieron todas las respuestas correctas demuestran diferentes dominios sobre lo que llamo capacidades avanzadas (esto también se observa, pero de forma menos intensa, en la corrección de las preguntas objetivas de discusión y en las objetivas).

Algunos alumnos, por ejemplo, demuestran tener conocimiento y comprensión de forma más amplia que otros, o bien expresan de manera más clara en sus respuestas que hicieron un análisis con criterio o una síntesis derivada de abstracciones ( $y$ no de hechos) cuya extensión es diferente a la de los otros. Finalmente podemios constatar que los juicios que realizan algunos alumnos están mejor fundamentados que los de otros.

Particularmente, pero no exclusivamente, en las preguntas de asociación de conocimientos, son utilizadas las estrategias de probar si el alumno manifestará o no comportamieuto de aplicación, relación entre contenidos o extrapolación (véanse preguntas 26,29 y 32 como ejemplos respectivos de estos aspectos). Esto sucede, pues de acuerdo con el modelo teórico expuesto, esas manifestaciones son consideradas vinculadas directamente al nivel de las capacidades avanzadas intermedias y finales. Por lo tanto, dentro de ese modelo de prueba, 
ese tipo de preguntas, además de ser capaz de suministrar alguna idea de las capacidades subyacentes de los alumnos, puede servir también como un indicador directo del desarrollo de las capacidades avanzadas.

Siendo así, principalmente a partir del análisis interpretativo de las respuestas a las preguntas de asociación de conocimiento, podemos colocar en evidencia si el alum. no presenta las capacidades de análisis, síntesis y evaluación de la forma en que fueron definidas en sentido estricto (en el nivel de las capacidades iniciales), o bien si estas capacidades están más desarrolladas (en el nivel de las capacidades avanzadas). De esa misma forma también podemos, a través del análisis directo de las respuestas a las preguntas de asociación de conocimiento, tener más indicios respecto al desarrollo de las demás capacidades avanzadas.

Obviamente el mérito no es exclusivo de este tipo de pregunta, puesto que incluso en las preguntas objetivas, que exigen una gran capacidad de síntesis, esto puede ser observado. Sin embargo, como la interpretación de qué capacidades -sean avanzadas, iniciales o subyacentesestán implícitas en una respuesta dada, es muy difícil, con este modelo de prueba, basado en el esquema de interacción entre capacidades, aquí presentado, tornarIas más explícitas. En el caso de la verificación de las capacidades avanzadas, esto puede realizarse a través de la comparación entre las respuestas a las preguntas de asociación de conocimientos con las demás respuestas referentes a otros tipos de preguntas.

\section{ALGUNAS LIMITACIONES DE ESTE MODELO DE PRUEBA}

\section{Modelo de interacción entre capacidades en proceso de perfeccionamiento}

La comprensión del modelo teórico que incluye la diferenciación y descripción de la interacción de las capacidades es muy importante para entender las posibles aplicaciones del modelo de prueba que presentamos. Esos modelos han sido, en parte, construidos a través del análisis interpretativo y criterial (acompañado de la diferenciación entre lo que son capacidades y manifestaciones de las capacidades), tanto en las preguntas de pruebas como en las respuestas que los alumnos dan a estas preguntas.

Debo destacar que estos modelos están siendo perfeccionados y se hace aún necesario un estudio mucho más profundo y detallado sobre la posible existencia de otras capacidades que aquí no fueron descritas, así como un estudio de qué cosas estarían influyendo en el surgimiento de las capacidades subyacentes presentadas (hecho que es de suma importancia para la actividad docente). También sería importante tener claro cuáles son las distintas "naturalezas», en términos de actividad mental, de las diferentes capacidades que discutí.
Para ilustrar rápidamente estos puntos véanse, por ejemplo, las dificultades que actualmente enfrento para interpretar el hecho de que algunos alumnos, en general los más reflexivos, incluso no revelando mucha comprensión y entendimiento, manifiestan indicios de las manifestaciones consideradas como típicas del nivel de las capacidades avanzadas iniciales. En principio creo que esos alumnos tal vez tengan una capacidad (que tal vez pudiese ser llamada de abstracción o reflexión) que debería ser colocada en el nivel subyacente, porque parece ocurrir en esos casos sin haber sido constatada muy claramente la manifestación de las capacidades avanzadas o alguna de las iniciales.

\section{LA VALIDEZ Y LA CONFIABILIDAD}

Actualmente estoy realizando pruebas con números iguales de los cinco tipos de preguntas que expuse y, después de corregirlas siguiendo una clave previa para garantizar la confiabilidad de los resultados, intento verificar si las notas que los alumnos obtuvieron tienen un signficado real (el antiguo problema de la validez de las pruebas escritas).

En este aspecto en particular aún no tengo conclusiones definitivas, aunque haya observado que, aparentemente, los alumnos que no muestran un buen desarrollo de las capacidades subyacentes no acostumbran a obtener una nota superior a 3 -en una escala de 0 a 10-. Alumnos que demuestran avances en estas capacidades, pero no de forma tan intensa como para presentar las manifestacio nes referentes a las capacidades avanzadas, no obtienen notas superiores a 565,5 .

Paradójicamente, otros hechos se manifestaron. En el transcurrir de los dos años eq que este modelo de prueba ha sido aplicado, se observó que alumnos que acostumbran obtener notas superiores a 5 , en pruebas que se refieren a ciertos contenidos, eventualmente al hacer pruebas que se referían a los mismos contenidos obtenían notas más bajas. En esos casos también pude constatar la falta de motivación y tenacidad de esos alumnos al realizar las pruebas en que obtuvieron notas más bajas que las esperadas, manifestada a través de preguntas respondidas a medias o con caligrafia revelando prisa al escribir; además no había borradores de las respuestas, o el propio alumno confesó que no se esmeró mucho en las respuestas.

De esta forma, obligatoriamente tengo que hacer constar que la evaluación hecha, considerando como única fuente de recolección de datos las pruebas escritas, puede suministrar algunas informaciones al profesor para retroalimentar el curso. Siendo esas pruebas escritas hechas según el modelo que propongo, creo que muchas más informaciones estarán al alcance del profesor en lo que se refiere específicamente al proceso de aprendizaje (enfocando particularmente la cuestion de las capacidades). Sin embargo, no debemos considerar el uso exclusivo de pruebas escritas como la única forma de obtener información sobre la actividad docente y de los alumnos 
-y así poder evaluarlas y retroalimentarlas. Varios métodos, como aquéllos presentados por Rodríguez y sus colaboradores (1992), deben ser utilizados; de hecho en este trabajo simplemente hemos presentado un modelo de prueba que intenta optimizar la recogida de datos a través de las pruebas escritas.

\section{COMENTARIOS FINALES}

\section{La no-consideración de las capacidades subyacentes en el uso de la taxonomía}

La diferenciación de los tipos de preguntas que aquí realizo no concuerda exactamente con la categorización de la taxonomia de Bloom, aunque he observado que existen algunas coincidencias. Muchas preguntas ultraobjetivas, por ejemplo, pueden ser realizadas para verificar el comportamiento de conocimiento; varias de las preguntas objetivas corresponden a preguntas para el comportamiento de síntesis; y algunas de las preguntas de asociación de conocimiento pueden ser consideradas como preguntas para el comportamiento de aplicación.

Fuera de esto, lo que observamos comúnmente en los ejemplos para un determinado tipo de pregunta que establecí es que podemos encontrar preguntas que corresponden a las diversas categorías de la taxonomía. Véase, por ejemplo, la pregunta 14, que podría ser una pregunta para el comportamiento de síntesis, en comparación con las preguntas 8 y 12. Estas dos últimas, evidentemente, según el contexto, podrian corresponder respectivamen te a preguntas para el comportamiento de aplicación y conocimiento. Sin embargo, dentro de lo que aquí presentamos, tanto la pregunta 14 como la 8 y la 12 son ejemplos de preguntas objetivas.

Siendo así, debemos aclarar la relación entre las categorías taxonómicas de Bloom y la categorización de las preguntas que aquí presentamos. A fin de cuentas, objetivos educativos, como, por ejemplo, la síntesis, el análisis y la evaluación, tan admirablemente presentados por Bloom (1956), parecen corresponder a las capacidades reales, siendo percibidas en diferentes tipos de preguntas que se preocupan por detectarlas. Sin embargo, creo que debemos identificar diferentes tipos de pregun " tas por el hecho de poder, no sólo reconocer los diferen. tes niveles de capacidades, sino, sobre todo, facilitar la recogida de datos en lo que se refiere específicamente a las capacidades más subyacentes. De esa manera, podemos, dar un mejor enfoque al processo de enseñanzaaprendizaje en sí y no únicamente a los resultados de comportamiento que pretendíamos observar en los alumnos al final de una unidad de estudios.

Con ello podemos hacer una pequeña reflexión inicial respecto al feedback que ese modelo de prueba, que tiene en consideración la existencia de capacidades subyacentes, puede dar, asî́ como al trabajo que el profesor puede realizar. Para aclarar esto, recordemos el modelo teórico de interacción entre capacidades presentado, a partir del cual podemos decir que el gran desarrollo de la capaci- dad de percepción particular, aunada al desarrollo de las capacidades de memoria y de expresión, contribuyen inmediatamente a la formación y desarrollo de dos capacidades más superyacentes a éstas: la de conocimiento y la de comprensión en un sentido estricto (dentro del nivel de las capacidades iniciales, véase la figura 1).

Teniendo esto en mente, no podemos imaginar que, por ejemplo, la comprensión en sentido estricto haya surgido de Ia nada. Ciertamente que esta capacidad sólo aparecerá como consecuencia del desarrollo de otras capacidades más subyacentes a ella, como las de percepción particular, expresión y memoria, las cuales a sus vez están contempladas dentro de las preguntas ultraobjetivas y objetivas, y pueden ser trabajadas directamente por el profesor en sus aulas.

Esas capacidades subyacentes se constituyen, en principio, como bases para ser reconocidas (particularmente con el uso de ese modelo de prueba) y trabajadas, promoviendo para que las capacidades menos subyacentes puedan erguirse realmente con solidez y consistencia.

\section{Algunos breves ejemplos de feedback referentes al proceso de enseñanza-aprendixaje}

A pesar de las limitaciones que mencionamos, sólo a través del análisis de pruebas escritas encuentro varias informaciones que han sustentado algunas reformas en el curso. Aquí resumiré dos breves ejemplos: uno se refiere al desarrollo de la capacidad de expresión (que, a pesar de haber sido facilitada con el uso de este modelo de prueba, podría haber ocurrido sin su utilización); y otro referente a la propia restructuración del curso (que sólo puede ocurrir después de adoptar este modelo de prueba).

Exclusivamente a partir de la falta de clatidad en las respuestas suministradas por los alumnos en los distintos tipos de preguntas, he identificado diferentes tipos de errores en la escritura, que influyen en la expresión de una idea. Una simple relación, sin mayores explicaciones, de esos «problemas de expresión escrita» es presentada en la figura 3 .

A partir de este análisis, en algunas aulas, comencé a intentar trabajar específicamente en uno u otro de estos aspectos. Además de eso, sólo después de realizar este análisis, tuve una mayor seguridad para realizar otros tipos de trabajos de manera más intensa con los alumnos: el hábito de volver a ver y corregir un texto escrito, y el uso adecuado de elementos de enlace (conjunciones u otros elementos que unan ideas dándoles una secuencia lógica y coherente).

Con esos trabajos, la expresión de los alumnos comenzó a ser más fluida en lo que se refería a la articulación de ideas sobre los contenidos; este hecho favoreció, por ejemplo, un gran desarrollo de las capacidades avanzadas (algo que evidentemente puede ser identificado con mayor facilidad al utilizar este modelo de prueba). 
Figura 3

Relación de los tipos de problemas en la expresión escrita observados en los textos de los alumnos.

a) Precisión lingüística.

b) Falta de vocablos adecuados.

c) Uso de vocablos inadecuados.

d) Uso inadecuado de ciertas expresiones y signos de puntuación.

e) Uso inadecuado de pronombres.

f) Repetición excesiva de los mismos términos.

g) Lagunas en medio del texto.

h) Manifestación de incoherencia y contrasentido.

i) Uso inadecuado de elementos de entace.

j) Uso incorrecto de los tiempos verbales.

Otro ejemplo de información respecto al curso, obtenido a través del análisis de las preguntas de tipo objetivo dentro de este modelo de prueba, puede ser observado en el análisis de la figura 4. Después de un año de trabajo fueron analizadas todas las preguntas objetivas (a exepción de las del tipo ensayo) de las pruebas realizadas por los alumnos de $5^{2}, 6^{\circ}$ y $7^{\circ}$ grados. A través de esos análisis quedó claro que el curso exigía muchas más explicaciones de los alumnos de $7^{\circledR}$ que de los de $5^{2}$, los cuales tenían su trabajo limitado a manifestar «procedimientos", cosa que casi no era exigida a los alumnos del $7^{2}$. A su vez los alumnos del $6^{2}$, en ese sentido, tenian en sus cursos exigencias iguales (procedimientos y explicaciones), pese a que también les fuesen solicitadas manifestaciones de «descripción». A partir de este análisis todo el curso pudo ser repensado, surgiendo cuestionamientos de cuáles deberían ser realmente las exigencias y los enfoques de trabajo más adecuados para cada uno de los grados.

Otros ejemplos de feedback, mucho más interesantes que esos, pueden ser aportados. Sin embargo, como el espacio aquí es limitado, ese tema merece ser tratado más profundamente en un texto separado. Por el momento prefiero finalizar el artículo comentando algunos beneficios directos, producto del uso de los modelos que hemos descrito.

\section{El modelo de prueba y de integración entre capacidades en la enseñanza de ciencias}

Siguiendo una visión actual de la enseñanza de ciencias como investigación (Gil, 1993), debemos trabajar con situaciones-problema, construcción de modelos e hipótesis explicativas, estrategias de elaboración e interpretación de pruebas de hipótesis y, finalmente, como en cualquier trabajo científico, con la comunicación de los resultados e ideas (incluyendo el surgimiento de nuevas hipótesis) fruto de este proceso.
Figura 4

Porcentaje de los tipos de preguntas objetivas realizadas cn los años 1992.93 por alumnos de $5^{2}, 6^{9}$ y $7^{2}$ grado.

\begin{tabular}{|lrrr|}
\hline & $5^{2}$ & $6^{2}$ & 70 \\
\hline Procecimiento & 54,5 & 23,5 & 4,3 \\
Definición & 27,3 & 23,5 & 17,4 \\
Descripción & 0,0 & 35,3 & 26,1 \\
Explicaction & 18,2 & 17,7 & 52,2 \\
& $(\%)$ & $(\%)$ & $(\%)$ \\
\hline
\end{tabular}

Observación: En estos datos no están incluidas las preguntas objetivas que exigen la realización de peq̨ueños ensayos.

No podemos ignorar que, para que un alumno camine ese «ciclo de investigación», debe desarrollar diferentes capacidades que le permitan tener estas diferentes manifestaciones: construir hipótesis, elaborar e interpretar experiencias, divulgar los resultados e ideas finales. Creo que en el caso de que un profesor, al tratar la enseñanza de ciencias como investigación, trabaje sólo con estas manifestaciones y no con lo que influye para que éstas ocurran no tendrá éxito.

Considerando el modelo de interacción entre capacidades que fue propuesto, se tiene idea de cuáles serían algunos de los elementos básicos, esto es, las capacidades que permean todo este proceso de investigación. Teniếndose en cuenta el análisis de pruebas individuales de los alumnos, hecho en el modelo presentado, un profesor obtiene elementos que le facilitan percibir qué capacidades están siendo desarrolladas por los alumnos.

Esto es posible pues, como en el modelo de prueba escrita que adopto, los diferentes tipos de preguntas requieren, para ser respondidas correctamente, el uso de diferentes capacidades; Ia comparación de las respuestas dadas por los alumnos y de la cantidad de respuestas correctas y de errores en la prueba como un todo (comparándose el rendimiento del alumno en los diferentes tipos de preguntas), nos puede ofrecer un cuadro diagnóstico preliminar del desarrollo de las capacidades de ese alumno.

Aún más, al hacer una prueba que se refiera a varios tipos de contenidos, con varias preguntas de cada uno de los tipos que aquí proponernos, el profesor puede empezar a diferenciar contenidos de habilidades específicas. Esto es común y de fácil diagnóstico cuando un alumno responde bien a las preguntas ultraobjetivas y objetivas y erra o no obtiene respuestas totalmente correctas en casi todas las preguntas objetivas de discusion, de discusión y argumentación y de asociación de conocimientos.

Como, en lo que se refiere a los diferentes asuntos, las preguntas ultraobjetivas y objetivas son correctas, podemos decir que, no las capacidades, sino las habilidades 
de memoria, expresión y percepción particular son satisfactorias. Respecto a la habilidad referente al criterio, además de ser fácilmente diferenciada, podríamos decir que, en un sentido general y no limitada a un contenido dado, debe ser trabajada en este alumno.

A partir de todo ello, queda claro que un cuadro de retroalimentación, y más específicamente en referencia a ciertos aspectos del proceso de enseñanza-aprendizaje -algo que es fundamental en una visión actualizada de la enseñanza de ciencias-, puede ser obtenido con más facilidad a través del uso del modelo de prueba y de interacción entre capacidades que presentamos que con la utilización de la taxonomía de Bloom.

\section{REFERENCIAS BIBLIOGRÁFICAS}

ALlAi, L., CARDINET, J. y PERRENOUD, P. (1986). A Avaliação Formativa num Ensino Diferenciado. Coimbra: Almedina.

BLOOM, .S., ENGELHART, M.D., FURST, E.J., HILL, W.H. y KRATHWOHL, D.R. (1973). Taxionomia de Objetivos Educacionais - Volume 1 Domino Cognitivo. Porto Alegre: Globo.

BLOOM, B.S., HASTINGS, J.T. y MADANS. G.F. (1971). Handbookon Formative and Summative Evaluation of Student Learning. Nueva York: McGraw.Hill.

\section{NOTA}

1 Fecha de la publicación póstuma de la obra cuya traducción a la lengua portuguesa, publicada en Brasil en 1987, es citada en la bibliografía.

GIL, D. (1993). Contribución de la histotia y de la filosofía de las ciencias al desanollo de un modelo de enseñanza/aprendizaje como investigación. Enseñanza de las Ciencias, 11(2), pp. 197-212.

RODRÍGUEZ BARREIRO, L.M., GUTIÉRREZ MUZQUIZ, F.A. y MOLEDO CEA, J. (1992). Una propuesta integral de evaltración en ciencia. Enseñanza de las Ciencias, 10(3), pp. 254-267.

VYGOTSKY, L.S. (1987). Pensamento e Línguagem. São Paulo: Martins Fontes. 


\section{ANEXO \\ EJEMPLOS DE LOS DIFERENTES TIPOS DE PREGUNTAS \\ EJEMPLO DE PREGUNTAS ULTRAOBJETIVAS}

1. Defina qué es pasteurización.

2. Asocie las columnas indicando a qué grupo de invertebrados pertenecen los siguientes seres vivos:
a. Cucaracha
b. Estrella de mar
c. Coral
d. Pulpo
e. Camarón
f. Escorpion
() Molusco
() Crustáceo
() Equinodermo
() Insecto
() Arácrido
() Cnidario

3. ¿Qué es abiogénesis?

4. ¿Cuál es la función de las hemacias?

5. Complete la tabla siguiente:

\begin{tabular}{|c|c|c|}
\hline Partícula atómica & Carga eléctrica & Masa \\
\hline & & \\
\hline & & \\
\hline & & \\
\hline
\end{tabular}

6. Indique en el dibujo el nombre de la parte del microscopio señalada por la flecha.

\section{EJEMPLO DE PREGUNTAS OBJETIVAS}

7. Describa cómo es una célula por dentro.

8. Haga una cadena alimenticia entre los siguientes seres vivos...

9. Usted está escribiendo un pequeño diccionario y debe elaborar definiciones precisas capaces de diferenciar cada uno de los cuatro términos, señalados a continuacion: a) relación sexual, b) unión sexual, c) sexo, d) reprodıcción.

10. ¿Qué es el mal de Chagas?

11. ¿Como pensaba Galeno que funcionaba el corazón?

12. Explique cómo se reproduce un virus.

13. Sabiendo que el dibujo es un esquema de una estación de tratamiento de agua, explique lo que ocurre en la parte señalada por la flecha.

14. Vamos a imaginar que usted fue invitado para escribir en un diario un pequeño artículo de exactamente seis líneas sobre los protozoos. En el espacio que hay a continuación escriba el texto que usted enviaría al editor del diario.

\section{EJEMPLO DE PREGUNTAS OBJETIVAS DE DISCUSIÓN}

15. ¿Cuál es la importancia del descubrimento de Ios fósiles?

16. En una conversación alguien le afirma que: «Las plaquetas no son las únicas responsables de la coagulación sanguínea». Explique por qué usted debe discutir lo que esa persona dijo.

17. En diferentes cavernas observamos varios animales: murciélagos, arañas y hasta peces. Muchos peces que viven en las cavernas tienen una característica peculiar al ser comparados con sus «hermanos» que viven fuera de las cavernas: no poseen ojos. Cómo explicaría usted la ausencia de ojos en esos peces, según la teoría de la selección natural.

18. Debemos hacer una sustracción entre el número de masa y el número de protones para descubrir la cantidad de neutrones existentes en un átomo. ¿Por qué debemos hacer esta sustracción en lugar de realizar una adictión, una división o una multiplicación?

19. ¿Qué conclusión podría kaber obtenido Miller de la experiencia que realizó? 


\section{EJEMPLO DE PREGUNTAS DE DISCUSIÓN Y ARGUMENTO}

20. «Entonces quiere decir que todas las cosas están formadas de una serie de espacios vacíos.» Ésa fue la conclusión que uno de los alumnos de $5^{0}$ grado expresó a sus colegas al final de una clase. ¿Usted cree que esta conclusión es correcta? Discuta y dé argumentos a favor o en contra de la conclusión de ese alumno.

21. Usted fue hasta otro planeta donde existen piedras que tienen la capacidad de reproducirse, o sea de tener piedras hijas. De vuelta al planeta Tierta, usted diría a sus colegas que estuvo en un planeta con o sin vida? Justifique su respuesta.

22. Durante una conferencia de la ECO-92 cierta persona afirmó que «las plantas no sólo extraen gas carbónico del medio ambiente, también aportan gas carbónico al medio ambiente». ¿Esta persona esłaba diciendo una cosa correcta o no? ¿Por qué?

23. Explique por qué usted está de actuerdo o no con esta frase: «Todos los seres vivos no vertebrados son invertebrados.» 24. ¿La expresión «el hombre desciende del mono» es correcta para explicar la idea de evolución humana basada en los fósiles? Justifique y discuta stu respuesta.

\section{EJEMPLO DE PREGUNTAS DE ASOCIACIÓN DE CONOCIMIENTO}

25. ¿Cómo haría usted para simular, dentro de un Iaboratorio, el ciclo del agua en la naturaleza?

26. Si los volcanes expelen «constantemente» el magma terrestre, ¿por qué éste nunca sa acaba?

27. Cómo explicaría usted la existencia de fósiles de seres marinos vivos en el alto de la cordillera de los Andes, situada en la costa oeste de América del Sur.

28. Explique de dónde vienen las «burbujas» de aire que encontramos en el pan.

29. ¿Cuál es la relación que podemos establecer entre las conclusionts a las que Ilegaron Redi y Miller en sus experimentos?

30. ¿Existe alguna relación entre células y átomos?

31. ¿Por qué el vídeo que usted vio sobre el desarrollo embrionario de las salamandras no podría ser hecho con mamíferos?

32. Si los átomos no pueden ser vistos, ¿cómo sabemos que existen? 
